\title{
Mood Scores in Relation to Hormone Replacement Therapies during Menopause: A Prospective Randomized Trial
}

\author{
Gogsen Onalan, ${ }^{1,2}$ Reside Onalan, ${ }^{3}$ Belgin Selam, ${ }^{4}$ Munire Akar, ${ }^{3}$ \\ ZiYa GunENC $^{1}$ and Ata TOPCUOGLU ${ }^{4}$ \\ ${ }^{1}$ Department of Obstetrics and Gynecology, Ankara Training and Research \\ Hospital, Ankara, Turkey, \\ ${ }^{2}$ Centrum Clinic, Ankara, Turkey, \\ ${ }^{3}$ Zekai Tahir Burak Women's Health and Education Hospital, Ankara, Turkey, and \\ ${ }^{4}$ Department of Obstetrics and Gynecology, Izzet Baysal Faculty of Medicine, \\ Abant Izzet Baysal University, Bolu, Turkey
}

Onalan, G., Onalan, R., Selam, B., Akar, M., Gunenc, Z. and Topcuoglu, A. Mood Scores in Relation to Hormone Replacement Therapies during Menopause: A Prospective Randomized Trial. Tohoku J. Exp. Med., 2005, 207 (3), 223-231 — There is lack of studies in literature about the long-term effects of hormone replacement therapies and cholesterol levels on mood scores in menopause. In the present study we have investigated whether serum lipid levels affect mood scores in menopause and evaluated the long-term effects of the combined hormone replacement regimens (HRT) on depressive symptoms in postmenopausal women. In this prospective-randomized, placebo-controlled, double-blind study, 286 women in menopause were divided into four groups according to therapeutic regimens they received; 1) Conjugated equine estrogen (CEE) of $0.625 \mathrm{mg}$ plus medroxyprogesterone acetate (MPA) of $2.5 \mathrm{mg}(n=79), 2)$ CEE $(0.625 \mathrm{mg})$ plus MPA of $5 \mathrm{mg}(n=$ $77), 3)$ tibolone of $2.5 \mathrm{mg}$ (a selective tissue estrogenic activity regulator) $(n=76)$, and 4$)$ Calcium (Ca) of 1,000 mg $(n=54)$. Beck Depression Inventory (BDI), and serum levels of lipoprotein lipids were assessed before and after 12-months of treatment with oral continuous HRT and Ca supplementation. BDI scores in the study groups were not correlated with lipid profiles. We compared two subgroups of patients with initial BDI scores 0-14 (normal mood scores) in order to asses for the possible relation between the lipid profile and mood. Following treatment, first subgroup had increased scores to 15-30 (mildly depressed women, $n=27$ ) and the second subgroup preserved BDI scores of 0-14 (normal mood scores, $n=23$ ). Serum levels of total cholesterol, high-density lipoprotein, lowdensity lipoprotein and body mass index were found to be similar between these two groups. BDI scores decreased significantly in all HRT groups after 12 months of treatment, compared to Ca group $(p<0.05)$. We did not observe any correlation between BDI scores and lipid profiles before and following continuous HRT or Ca supplementation. Continuous combined hormone replacement regimens, CEE + MPA and tibolone, have superior long-term effects on mood scores in menopause and should be considered during

Received June 16, 2005; revision accepted for publication September 1, 2005.

Correspondence: Gogsen Onalan, M.D., Centrum Clinic, Nenehatun, No: 59 GOP, Ankara 06700, Turkey.

e-mail: gogsenonalan@yahoo.com 
the decision process for use of HRT due to menopausal symptoms. - menopause; cholesterol; depression; HRT

(C) 2005 Tohoku University Medical Press

Epidemiologic studies worldwide report the prevalence of major depressive disorder about twice as high in women compared to those in men, especially during the childbearing years. Neuromodulatory effects of estrogen and other gonadal steroids impose a risk factor for mood disorders in women due to differential sensitivity to physiological hormonal fluctuations during premenstrual, postpartum periods, and perimenopause (Barnhart et al. 1995 ; Joffe and Cohen 1998; van Dam et al. 1999). Response to antidepressant therapy also differs in premenopausal and postmenopausal women proposing sex steroid hormones as an etiologic factor (Kornstein 1997). Neurobiological studies demonstrate that estrogen regulates numerous aspects of noradrenergic, serotonergic, GABAergic and dopaminergic transmission and may substantiate mood and depressive states (Huttner and Shepherd 2003). Estrogen augments serotonin concentrations at synapses in postmenopausal women (Halbreich 1997). Serum levels of serotonin positively correlate with serum levels of estradiol in postmenopausal women and decreased levels of serotonin in the peripheral circulation and brain can be reversed by hormone replacement therapy (HRT) (Halbreich 1997).

Cholesterol is another modulatory factor in serotoninergic pathway (Brunner et al. 2002). In vitro and animal studies report modulation of central serotonergic neurotransmission by cholesterol content in neuronal membranes (Shih and Ohsawa 1983; Kaplan et al. 1994). Recent epidemiologic and clinical studies investigating the relationship between serum cholesterol levels and depressive mood have yielded equivocal results (Glueck et al. 1994; Boston et al. 1996; Huang et al. 2003; Ledochowski et al. 2003; Nakao and Yano 2004). In general, there has not been any consensus whether high or low cholesterol levels are associated with depression.

The relationship between menopause and de- pression is not clearly defined. However, clinical studies report frequent decline in cognitive functions such as memory, altered mood and reduced libido in women during menopause (Halbreich 1997; Genazzani et al. 2002). Prospective population-based studies report mild depression/mood changes in perimenopausal women rather than accusing menopause as a major etiology for depressive symptoms (Bungay et al. 1980; Hunter 1992). However, a recent meta-analysis revealed that unopposed estrogen most effectively improved depressed mood among postmenopausal women (Zweifel and O'Brien 1997). Therefore, it is possible that the use of HRT in postmenopausal women moderates the relation between depression and cholesterol, and could ameliorate the negative associations previously found. HRT in postmenopausal women has also been associated with a reduction in total cholesterol and low-density lipoprotein cholesterol and an increase in high-density lipoprotein cholesterol (Guetta and Cannon 1996).

To our knowledge, long-term follow-up studies about mood scores in relation to both HRT and cholesterol levels are lacking in menopause. In the present study we aimed to determine if lower serum levels of lipids were risk factors for elevated depression scores, and whether long-term continuous combined HRT affected mood scores in postmenopausal women.

\section{Materials and Methods}

The study was conducted in the menopause clinic of Ankara Research and Training Hospital between 1999 and 2001. Three hundred thirty two women out of 1,215 women attending the menopause clinic were included in the study.

\section{Inclusion criteria}

Inclusion criteria for the study were; physiologic menopause with amenorrhea for at least 1 year and follicle stimulant hormone (FSH) levels $\geqq 35 \mathrm{mIU} / \mathrm{ml}$. 
Patients with premature ovarian failure, surgical menopause, diabetes mellitus, hypertensive disease, previous breast or endometrial cancer and patients using anticonvulsive, antidepressive, antilipidemic drugs and drugs affecting lipid levels (e.g., betablockers, diuretics, androgens, disulfram, corticosteroids, levadopa, and aminosalycylic acid) were excluded from the study. All subjects were randomized according to computer-blocknumber by a block design of 20 . The informed consent from all subjects and the local ethics committee approval were obtained. The current study was approved by Ethics Committee of Ankara Training and Research Hospital.

Patients were free of low-calorie, cholesterol-lowering diets, alcohol and were nonsmokers. Blood samples were drawn after a night of fasting. Automated enzymatic calorimetric method was used for cholesterol and triglyceride determinations (Reflotron ${ }^{\circledR} 2000$, Roche, Mannheim, Germany)

\section{Therapeutic regimens}

Patients who agreed to use HRT were randomized into one of the three oral continuous HRT protocols. The regimens were daily course of either conjugated equine estrogen (CEE) $0.625 \mathrm{mg}$ plus medroxyprogesterone acetate (MPA) $2.5 \mathrm{mg}$ (Premelle 2.5 ${ }^{\circledR}$; Wyeth, Istanbul, Turkey) $(n=79)$, CEE $0.625 \mathrm{mg}$ plus MPA $5 \mathrm{mg}$ (Premelle $5^{\circledR}$; Wyeth) $(n=77)$, or tibolone $2.5 \mathrm{mg}$ (a steroid compound that combines oestrogenic, progestogenic and androgenic properties that mimic the action of the sex hormones, Livial ${ }^{\circledR}$; Organon, Istanbul, Turkey) ( $n=$ 76) for 12-months of duration. An additional group of patients who only agreed to use medications other than hormones were prescribed calcium supplementation, Calcium Sandoz Forte 1,000 mg (Roche, Istanbul, Turkey) $(n=54)$. Patients in HRT group were not subscribed calcium during the study period in order to evaluate the effects of HRT and calcium treatments separately.

\section{Study parameters}

Studied parameters were recorded before and following 12 months of treatment period Patients were evaluated as their own controls. Body mass index (BMI) was measured as $\mathrm{kg} / \mathrm{m}^{2}$. Patients' educational, marital, socio-economic status and history of premenstrual syndrome (PMS), postpartum depression were recorded. Postpartum depression was defined as postpartum onset of reduction in mood, performance and drive as well as feelings of hopelessness usually accompanied by addi- tional feelings of reduced self esteem, guilt, suicidal thoughts, changes of appetite, weight, libido, psychomotor activity and sleep, all lasting most of the time for at least two weeks (Sampson 1989). PMS was defined as symptoms that improve with either at the onset of menstruation or shortly after (American Psychiatric Association 1994). Prior history of PMS and postpartum depression diagnosis were recorded from the previous records of the patients (total cholesterol [TC], high density lipoprotein [HDL], low density lipoprotein [LDL] and triglyceride [TG] levels were measured before and after the treatment period).

\section{Psychometric assessments}

Psychometric assessments of depressive symptoms were performed with the translated version of the Beck Depression Inventory (BDI) which was tested and approved for the Turkish population (Hisli 1988). BDI with self rating scales of 21 items (each item rated 0 to 3 ) provides a quantitative assessment of the intensity of depression over the past week (Beck et al. 1961). In the current study BDI scores were recorded as 0 to 63 and women with scores $0-14$ were accepted as normal while those with scores of 15-30 were accepted as mildly depressed.

\section{Statistical analysis}

Normality assumption was met for all continuous variables to be used in the regression analyses with the exception of HDL. Analyses were therefore conducted with logarithmic transformed data for this measure. Primary data analyses were conducted in the following manner; first, zero-order correlations were computed among all variables of interest. Next, hierarchical regression analyses were used to examine the contributions of BDI and HRT status in predicting lipid levels, after controlling for the effects of age, BMI, and HRT status. Data were reported as mean \pm S.D. Numerical data were normally distributed (tested by Kolmogorov-Smirnov test). Categorical data were analyzed using chi-square and numerical data were analyzed using student's test (two-tailed) and one way ANOVA. Repeated measures of analysis of variance were used for comparisons of treatments at 6-12 months. $P$ value $<0.05$ was considered to be statistically significant. Statistical analyses were performed using the SPSS for windows version 10. 


\section{Results}

Initial number of subjects and patients that were followed up by the end of the study were 332 and 286, respectively. Forty-one patients from HRT group (18\%) and $5(9 \%)$ patients from calcium treatment group dropped out from the study.

Baseline parameters were similar between HRT and calcium treatment groups $(p>0.05)$ (Table 1). Patients' baseline BDI scores were mildly elevated in all study groups. Average age for menopause was $52.4 \pm 5.9$ among women participating in the current study.

BDI scores in the study groups were not correlated with lipid profiles, history of PMS, postpartum depression, age of husband, socioeconomic and educational levels $(p>0.05)$.

We did not observe any significant differences in serum levels of TC, HDL, LDL, TG and BMI values among patients with BDI scores of 0-14 and 15-30 before and after treatment. BDI values did not differ among patients with hypo- cholesterolemia (TC $<160 \mathrm{mg} / 100 \mathrm{ml}, n=25$ ), normocholesterolemia $(\mathrm{TC}=160-220 \mathrm{mg} / 100 \mathrm{ml}$, $n=150$ ) or hypercholesterolemia (TC $>220$ $\mathrm{mg} / 100 \mathrm{ml}, n=107)$ and also with serum levels of HDL $\geqq 35$ or $<35 \mathrm{mg} / 100 \mathrm{ml}(p>0.05)$.

We compared two subgroups of patients with initial BDI scores of 0-14 (normal mood scores). Following treatment, first subgroup had increased scores to 15-30 (mildly depressed women, $n=27$ ) and the second subgroup preserved BDI scores of 0-14 (normal mood scores, $n=23$ ). We did not observe any significant differences related to serum levels of TC, HDL, LDL and BMI values between the two subgroups $(p>0.05)$ (Table 2).

We analyzed the differences about lipid profiles before and following 12 months of treatments in each study group. TC, LDL levels and BDI scores were significantly different in all HRT groups compared to those in the Ca group ( $p<$ 0.05 ), however differences in HDL levels were significantly different among all groups ( $p<0.05$ ). BMI values among the HRT groups were not significantly different before and after 12 months of

TABLE 1. Baseline parameters of the study groups

\begin{tabular}{lcccc}
\hline \multicolumn{1}{c}{ Parameter } & $\begin{array}{c}\text { CEE + MPA 2.5 mg } \\
(n=79)\end{array}$ & $\begin{array}{c}\text { CEE + MPA 5 mg } \\
(n=77)\end{array}$ & $\begin{array}{c}\text { Tibolone 2.5 mg } \\
(n=76)\end{array}$ & $\begin{array}{c}\text { Calcium 1,000 mg } \\
(n=54)\end{array}$ \\
\hline Age (years) & $51.6 \pm 6$ & $52.5 \pm 6$ & $53.3 \pm 6$ & $52.4 \pm 5.9$ \\
BMI (kg/m $\left.{ }^{2}\right)$ & $25.7 \pm 3.5$ & $25 \pm 4.4$ & $25 \pm 3.7$ & $25.8 \pm 3.6$ \\
Number of children & $2.1 \pm 0.1$ & $1.1 \pm 0.1$ & $1.1 \pm 0.1$ & $1.3 \pm 0.1$ \\
Marital status & & & & \\
$\quad$ Married (\%) & 83 & 87 & 86 & 84 \\
$\quad$ Single (\%) & $6(5)$ & $5(4)$ & $5(4)$ & $6(3)$ \\
$\quad$ Widow (\%) & $11(9)$ & $7.8(6)$ & $9.2(7)$ & $10(5)$ \\
Menopause duration (years) & $4.4 \pm 4$ & $4.1 \pm 3.8$ & $5.1 \pm 4.3$ & $4.7 \pm 3.3$ \\
Beck depression inventory & $20.9 \pm 13.8$ & $17.8 \pm 12.8$ & $19.3 \pm 13.5$ & $16.2 \pm 9.3$ \\
PMS (\%) & 49 & 58 & 59.2 & 51 \\
Postpartum depression (\%) & 8.8 & 6.4 & 6.5 & 5.4 \\
TC (mg/100 ml) & $210.2 \pm 35$ & $211.2 \pm 39.3$ & $218.5 \pm 38.4$ & $214.7 \pm 43.7$ \\
HDL (mg/100 ml) & $45.8 \pm 35.4$ & $46.1 \pm 14.8$ & $48 \pm 13.3$ & $45.4 \pm 13.3$ \\
LDL (mg/100 ml) & $137.9 \pm 36.6$ & $138.7 \pm 38.3$ & $144.8 \pm 38.4$ & $141.1 \pm 43.7$ \\
TG (mg/100 ml) & $128.3 \pm 85.6$ & $127.7 \pm 59$ & $139.8 \pm 74.6$ & $121.3 \pm 80.6$ \\
\hline
\end{tabular}

Values are mean \pm S.D. unless otherwise indicated. Differences among the groups were not statistically significant $(p>0.05)$. BMI, body mass index; PMS, Premenstrual syndrome; TC, total cholesterol; HDL, high density lipoprotein; LDL, low density lipoprotein; TG, triglyceride. 
TABLE 2. Mean serum lipid levels in two subgroups of patients with initial BDI scores of $<15$ before treatment

\begin{tabular}{lccl}
\hline & $\begin{array}{c}\text { Patients with BDI scores of }<15 \\
\text { before treatment, and } \geqq 15 \text { after treatment } \\
(n=27)\end{array}$ & $\begin{array}{c}\text { Patients with BDI scores of }<15 \\
\text { before and after treatment } \\
(n=23)\end{array}$ & $p$ value \\
\hline $\mathrm{TC}(\mathrm{mg} / 100 \mathrm{ml})$ & $213.4 \pm 37.8$ & $209.5 \pm 41.7$ & 0.08 \\
$\mathrm{HDL}(\mathrm{mg} / 100 \mathrm{ml})$ & $46.1 \pm 14.8$ & $47.3 \pm 15$ & 0.5 \\
$\mathrm{LDL}(\mathrm{mg} / 100 \mathrm{ml})$ & $143 \pm 77.9$ & $131.9 \pm 34.2$ & 0.2 \\
$\mathrm{BMI}\left(\mathrm{kg} / \mathrm{m}^{2}\right)$ & $25 \pm 3.7$ & $25.1 \pm 3.6$ & 0.5 \\
\hline
\end{tabular}

Values are mean \pm S.D. unless otherwise indicated. Differences among the groups were not statistically significant $(p>0.05)$. BMI, body mass index; TC, total cholesterol; HDL, high density lipoprotein; LDL, low density lipoprotein.

TABLE 3. Standardized regression coefficients, significance levels, and squared semipartial correlation for predictor variables

\begin{tabular}{lccccccccccccc}
\hline & \multicolumn{3}{c}{ Cholesterol } & \multicolumn{3}{c}{ Triglyceride } & \multicolumn{3}{c}{ HDL-C } & \multicolumn{3}{c}{ LDL-C } \\
\hline Predictor & $\beta$ & $p$ & $S r^{2}$ & $\beta$ & $p$ & $S r^{2}$ & $\beta$ & $p$ & $S r^{2}$ & $\beta$ & $p$ & $S r^{2}$ \\
Age & 0.17 & $<0.01$ & 0.03 & 0.02 & 0.62 & $<0.01$ & -0.03 & 0.29 & $<0.01$ & 0.1 & 0.02 & 0.2 \\
BMI & 0.49 & $<0.01$ & 0.24 & 0.19 & 0.01 & 0.04 & -0.06 & 0.57 & $<0.01$ & 0.4 & $<0.01$ & 0.16 \\
HRT & 0.02 & 0.77 & $<0.01$ & 0.01 & 0.9 & $<0.01$ & 0.07 & 0.34 & $<0.01$ & -0.05 & 0.43 & $<0.01$ \\
BDI & 0.009 & 0.85 & $<0.01$ & -0.02 & 0.67 & $<0.01$ & 0.07 & 0.25 & $<0.01$ & 0.023 & 0.68 & $<0.01$ \\
BDI $\times$ HRT & 0.01 & 0.82 & $<0.01$ & -0.08 & 0.25 & $<0.01$ & 0.09 & 0.23 & $<0.01$ & 0.004 & 0.95 & $<0.01$ \\
& $R^{2}$ & $F$ & $p$ & $R^{2}$ & $F$ & $p$ & $R^{2}$ & $F$ & $p$ & $R^{2}$ & $F$ & $p$ \\
Full model $^{*}$ & 0.28 & 22.2 & $<0.01$ & 0.44 & 2.6 & 0.026 & 0.016 & 0.9 & 0.4 & 0.18 & 12.9 & $<0.001$ \\
\hline
\end{tabular}

"Predictors: (Constant), BMI, age, HRT status, BDI, BDI $\times$ HRT.

treatment $(p>0.05)$.

Four regression analyses, one with each lipid measure (CHOL, HDL-C, LDL-C, and TG) as criterion, were conducted using the following predictor variables: age, BMI, HRT, BDI, and the interaction of HRT and BDI (Table 3). The model explained $28 \%, 4 \%, 1 \%$, and $18 \%$ of the variance in total cholesterol, TG, HDL-C and LDL-C, respectively $(p<0.05)$. Standardized betas, squared semi partial correlation coefficients, and significance values for the individual predictors included in each regression analyses by criterion variable are displayed in Table3. After removing the effects of all other predictors, the amount of shared variance between BDI scores and total cholesterol, TG, and LDL-C were $<1 \%$, respectively $(p>0.05)$. In addition, HRT and BDI interaction uniquely accounted for $<1 \%$ of the variance in all lipids.
Patients with elevated BDI scores ( $\geqq 15$ ) before treatment, and decreased BDI scores $(<15)$ after treatment were considered to have improved BDI scores. A multivariate logistic regression model was established in which "improvement in BDI scores" was considered as dependent variable whereas, baseline age, BMI, number of children, marital status, duration of menopause, premenstrual symptoms, postpartum depression, serum levels of total cholesterol, HDL, LDL and triglycerides and groups were considered as candidate predictive variables. We investigated baseline parameters that could predict "improvement in BDI scores" after treatment. There was a negative correlation between improved BDI scores and duration of menopause (odds ratio $=2.3,95 \%$ confidence interval 1.2-7, $p=0.002$ ) which also predicted the "responders" in the study. Each one year increase in duration of menopause was asso- 
Total Cholesterol

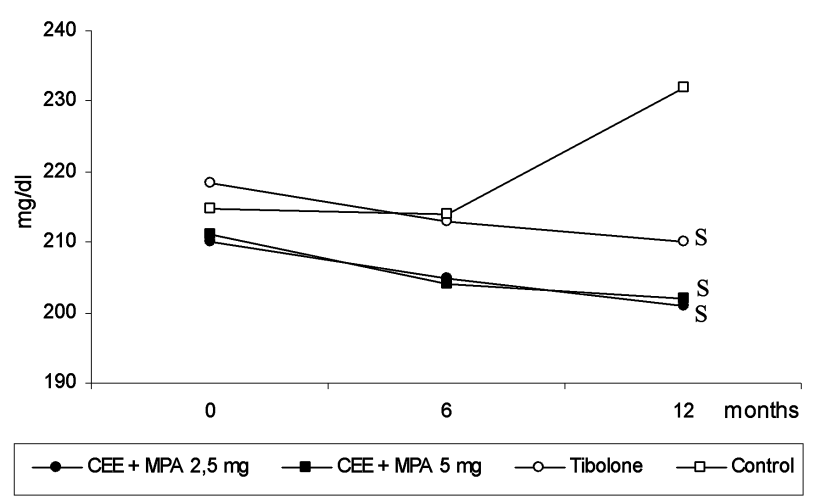

LDL

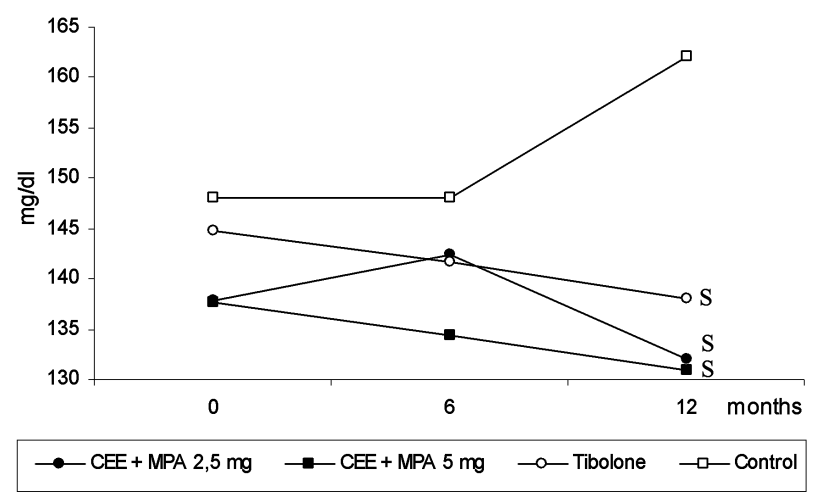

HDL

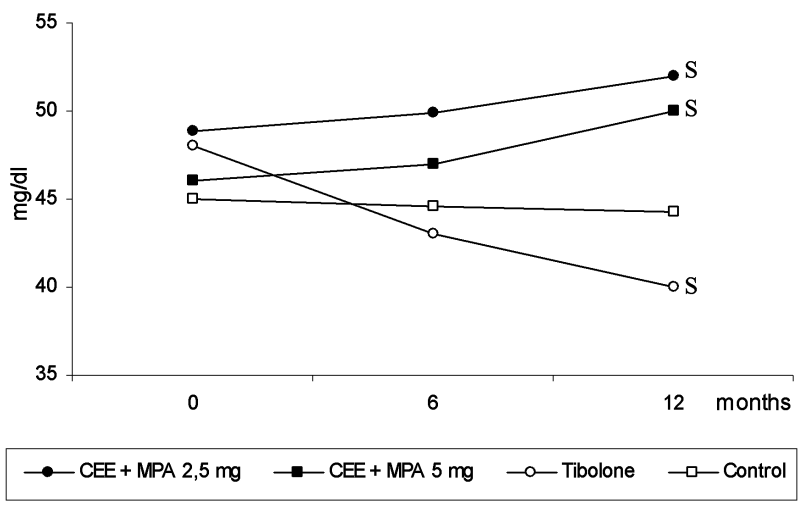

BDI

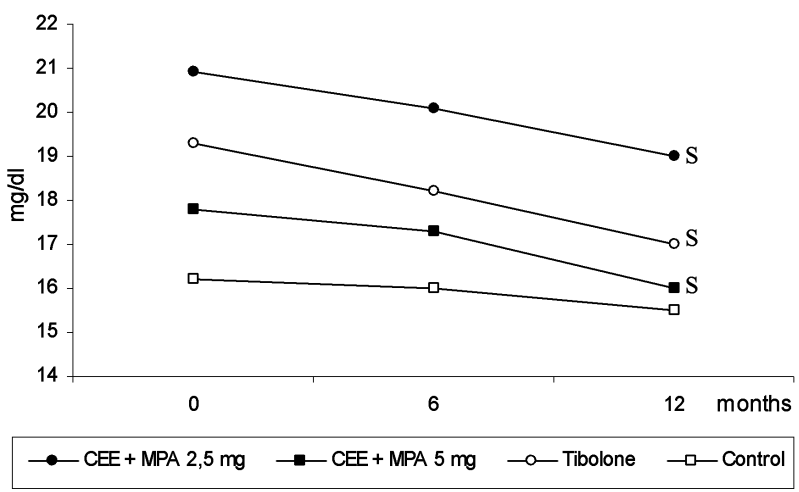

Fig. 1. Serum levels of total cholesterol, LDL, HDL and BDI scores before and after 6-12 months of calcium supplementation and HRT treatments including CEE $0.625 \mathrm{mg}$ plus MPA 2.5 mg, CEE 0.625 mg plus MPA $5 \mathrm{mg}$ and tibolone in menopause. TC, total cholesterol (mg/100 ml); LDL, low density lipoprotein $(\mathrm{mg} / 100 \mathrm{ml})$; HDL, high density lipoprotein $(\mathrm{mg} / 100 \mathrm{ml})$; BDI, beck depression inventory scores; CEE, conjugated equine estrogen; MPA, medroxyprogesterone acetate; S, statistically significant $(p<0.05)$. Differences among all subgroups were statistically significant $(p<0.001)$. Time-course differences were also statistically significant in each HRT group $(p<0.05)$.

ciated with 2.3 times of decrease in BDI scores. Decrease in basal BDI scores after 12 months of treatment was significant in all HRT groups compared to Ca group $(p<0.05)$.

\section{Discussion}

Our data evaluating the effect of cholesterol levels on BDI scores during menopause indicate that there is no correlation between BDI scores and lipid profiles before and following treatment with all regimens. Cholesterol levels do not introduce an additional risk factor for depressive disorder during menopause. When we analyzed our data with univariate statistical methods there were no relation between BDI score and lipid levels. Next when we used multiple lineer regression analyses, we also could not find any relation. Moreover, BDI scores in menopause are not related to history of PMS and postpartum depression, marital, educational and socioeconomic status of women in the study.

In the literature, only one study evaluated the potential interactive relationship of depressive symptomatology (that was assessed using the Center for Epidemiological Studies Depression Scale) and HRT status (that study did not take into account route, dose and duration), to lipoprotein lipids in 70 postmenopausal women. It reported 
an association between naturally occurring low cholesterol and higher levels of depressive symptoms (Brown et al. 2004). Interpretation of these findings should be made cautiously, because of the relatively small number of participant women and a cross-sectional design, which precludes conclusions regarding the directionality of association. This is in contrast to our prospective, randomized, placebo controlled study.

According to a previous report, $65 \%$ of the women who attend menopause clinics are within the depressed range of the Zung self-rating depression scale (Anderson et al. 1987). However, several studies report that women on HRT are different in many aspects from those of nonusers. Women who intend to use HRT have a more negative attitude toward menopause, lower levels of self-esteem, and higher levels of depressed mood and anxiety (Hunter and Liao 1994). Women willing to use HRT during menopause may receive higher benefits in mood. Therefore estrogen treatment, even in non-depressed individuals, enhances platelet ${ }^{3} \mathrm{H}$-imipramine binding and improves BDI scores (Sherwin and SuranyiCadotte 1990). Psychological functioning improves in asymptomatic peri-and postmenopausal women undergoing estrogen therapy (Ditkoff et al. 1991). Short term continuous hormone replacement therapy regimens improve mood scores, however there are no enough data about the long-term follow-up of these regimens in literature (Bukulmez et al. 2001).

In the current study we evaluated the longterm effects of continuous combined HRT regimens, tibolone and calcium supplementation on BDI scores in menopause. Calcium supplemented group was used as control simulating placebo effect for assessment of mood scores. Calcium supplemented group consisted of women who fulfilled all the inclusion criteria except that they were not willing to use HRT. Baseline parameters of this group were not different from those of three HRT groups. Following 12 months of treatment basal BDI scores decreased significantly in all HRT groups compared to those of Ca supplementation group. Our data confirms previous reports suggesting that estrogen replacement ther- apy is superior to placebo for mood disorders associated with hormonal fluctuations like postpartum depression, mild depressive diseases during perimenopause and menopause (Zweifel 1997; Schmidt et al. 2000; Soares et al. 2001; Grigoriadis and Kennedy 2002).

Recent data from the Women's Health Initiative (WHI) reports that HRT does not have a significant role for enhancing mood, quality of life and a variety of psychometric measures (Hays et al. 2003). However, WHI has been criticized as not being a primary prevention trial. Mean age of women in WHI study is above 60 years and most of the women have already been in menopause for 10 years. Following arguments about WHI study, use of HRT is suggested only for transitional vasomotor symptoms and genital atrophy during the first 2-3 years of menopause (Solomon and Dluhy 2003). HRT may also be valuable for symptoms of mild depression during this period of menopause.

Continuous CEE + MPA $2.5 \mathrm{mg}$ or $5 \mathrm{mg}$ and tibolone regimens may be expected to reduce the estrogenic effects by antagonistic activity. Progestins may have variable effects on the brain, occasionally inducing dysphoric mood and altered behavior (Genazzani et al. 2002). Especially by doubling doses of MPA we may expect lower BDI scores in menopause (Holst et al. 1989). However, we observed that the addition of MPA in two commonly prescribed doses as continuous combined HRT regimens did not significantly impair favorable effects of estrogen on the depressive symptoms and both combined regimens improved BDI scores evenly. Improvement in mood scores with continuous combined HRT may reflect the direct or indirect interactions of HRT by serotoninergic system. In addition, tibolone improves depressive symptomatology as much as CEE + MPA treatment. Tibolone and its metabolites may also act through estrogen receptors to enhance similar serotoninergic effects in the central nervous system.

DSM-IV standardized diagnostic criteria are used for confirming the diagnosis of major depression and can only be evaluated by an experienced psychologist or psychiatrist (American 
Psychiatric Association Diagnostic and Statistical Manual of Mental Disorders 1994). In the currents study we preferred widely used BDI that detects depressive symptoms, assesses the severity of depression and also evaluates the effectiveness of therapy in ameliorating the symptoms (Gath 1998). BDI has high test-retest reliability and moderate sensitivity to change over time, however it may have some criteria overlap as loss of appetite and weight are characteristic symptoms of both physical and psychological disorders. Good normative data are also available for BDI and scale provides a scheme for sub-dividing depressive symptoms as mild, moderate and severe.

We conclude that, BDI scores in menopause may not be correlated with lipid profiles before and following continuous HRT and Ca supplementation. Cholesterol levels may not introduce an additional risk factor for depressive disorder whereas continuous combined hormone replacement regimens including CEE + MPA and tibolone may improve mood scores long-term in menopause.

\section{References}

American Psychiatric Association Diagnostic and Statistical Manual of Mental Disorders (1994) DSM-IV 4th ed., American Psychiatric Association, Washington, DC, USA.

Anderson, E., Hamburger, S., Liu, J.H. \& Rebar, R.W. (1987) Characteristics of menopausal women seeking assistance. Am. J. Obstet. Gynecol., 156, 428-433.

Barnhart, K.T., Freeman, E.W. \& Sondheimer, S.J. (1995) A clinician's guide to the premenstrual syndrome. Med. Clin. North Am., 79, 1457-1472.

Beck, A.T., Ward, C.H., Mendelson, M., Mock, J. \& Erbaugh, J. (1961) An inventory for measuring depression. Arch. Gen. Psychiatry., 4, 561-571.

Boston, P.F., Dursun, S.M. \& Reveley, M.A. (1996) Cholesterol and mental disorder. Br. J. Psychiatry., 169, 682-689.

Brunner, J., Parhofer, K.G., Schwandt, P. \& Bronisch, T. (2002) Cholesterol, essential fatty acids, and suicide. Pharmacopsychiatry., 35, 1-5.

Bukulmez, O., Al, A., Gurdal, H., Yarali, H., Ulug, B. \& Gurgan, T. (2001) Short-term effects of three continuous hormone replacement therapy regimens on platelet tritiated imipramine binding and mood scores: a prospective randomized trial. Fertil Steril., 75, 737-743.

Brown, A.R., Giggey, P.P., Dennis, K.E. \& Waldstein, S.R. (2004) Depression and lipoprotein lipids in healthy postmenopausal women the moderating effects of hormone replacement therapy. J. Psychosom. Res., 56, 171-176.

Bungay, G.T., Vessey, M.P. \& McPherson, C.K. (1980) Study of symptoms in middle life with special reference to the menopause. Br. Med. J., 281, 181-183.

Ditkoff, E.C., Crary, W.G., Cristo, M. \& Lobo, R.A. (1991) Estrogen improves psychological function in asymptomatic postmenopausal women. Obstet. Gynecol., 78, 991-995.

Gath, D. (1998) The assessment of depression in perimenopausal women. Maturitas, 29, 33-39.

Genazzani, A.R., Monteleone, P. \& Gambacciani, M. (2002) Hormonal influence on the central nervous system. Maturitas, 43, Suppl. 1, S11-S17.

Glueck, C.J., Tieger, M., Kunkel, R., Hamer, T., Tracy, T. \& Speirs, J. (1994) Hypocholesterolemia and affective disorders. Am. J. Med. Sci., 308, 218-225.

Grigoriadis, S. \& Kennedy, S.H. (2002) Role of estrogen in the treatment of depression. Am. J. Ther., 9, 503-509.

Guetta, V. \& Cannon, R. (1996) Cardiovascular effects of estrogen and lipid lowering therapies in postmenopausal women. Circulation, 93, 1928-1937.

Halbreich, U. (1997) Role of estrogen in postmenopausal depression. Neurology, 48, S16-S19.

Hays, J., Ockene, J.K., Brunner, R.L., Kotchen, J.M., Manson, J.E., Patterson, R.E., Aragaki, A.K., Shumaker, S.A., Brzyski, R.G., La Croix, A.Z., Granek, I.A. \& Valanis, B.G. (2003) Women's Health Initiative Investigators. Effects of estrogen plus progestin on health-related quality of life. $N$. Engl. J. Med., 348, 1839-1854.

Hisli, N. (1988) Beck Depresyon Envanterinin gecerliligi uzerine bir calisma (A study on the validity of Beck Depression Inventory.) Psikoloji Dergisi., 6, 118-122.

Holst, J., Backstrom, T., Hammarback, S. \& von Schoultz, B. (1989) Progestogen addition during estrogen replacement therapy-effects on vasomotor symptoms and mood. Maturitas, 11, 13-20.

Huang, T.L., Wu, S.C., Chiang, Y.S. \& Chen, J.F. (2003) Correlation between serum lipid, lipoprotein concentrations and anxious state, depressive state or major depressive disorder. Psychiatry Res., 118, 147-153.

Hunter, M. (1992) The south-east England longitudinal study of the climacteric and postmenopause. Maturitas, 14, 117-126.

Hunter, M.S. \& Liao, K.L. (1994) Intentions to use hormone replacement therapy in a community sample of 45 -year-old women. Maturitas, 20, 13-23.

Huttner, R.P. \& Shepherd, J.E. (2003) Gonadal steroids, selective serotonin reuptake inhibitors, and mood disorders in women. Med. Clin. North Am., 87, 1065-1076.

Joffe, H. \& Cohen, L.S. (1998) Estrogen, serotonin, and mood disturbance: where is the therapeutic bridge? Biol. Psychiatry., 44, 798-811.

Kaplan, J.R., Shively, C.A., Fontenot, M.B., Morgan, T.M., Howell, S.M., Manuck, S.B., Muldoon, M.F. \& Mann, J.J. (1994) Demonstration of an association among dietary cholesterol, central serotonergic activity, and social behavior in monkeys. Psychosom. Med., 56, 479-484.

Kornstein, S.G. (1997) Gender differences in depression: implications for treatment. J. Clin. Psychiatry., 58, Suppl. $15,12-18$

Ledochowski, M., Murr, C., Sperner-Unterweger, B., Neurauter, G. \& Fuchs, D. (2003) Association between increased serum cholesterol and signs of depressive mood. Clin. Chem. Lab. Med., 41, 821-824.

Nakao, M. \& Yano, E. (2004) Relationship between major depression and high serum cholesterol in Japanese men. Tohoku J. Exp. Med., 204, 273-287.

Sampson, G.A. (1989) Premenstrual syndrome. Baillieres 
Clin. Obstet. Gynaecol., 3, 687-704.

Schmidt, P.J., Nieman, L., Danaceau, M.A., Tobin, M.B., Roca, C.A., Murphy, J.H. \& Rubinow, D.R. (2000) Estrogen replacement in perimenopause-related depression: a preliminary report. Am. J. Obstet. Gynecol., 183, 414-420.

Sherwin, B.B. \& Suranyi-Cadotte, B.E. (1990) Up-regulatory effect of estrogen on platelet $3 \mathrm{H}$-imipramine binding sites in surgically menopausal women. Biol. Psychiatry., 28, 339-348.

Shih, J.C. \& Ohsawa, R. (1983) Differential effect of cholesterol on two types of 5-hydroxytryptamine binding sites. Neurochem. Res., 8, 701-710.
Soares, C.N., Almeida, O., Joffe, H. \& Cohen, L.S. (2001) Efficacy of estradiol for the treatment of depressive disorders in perimenopausal women. Arch. Gen. Psychiatry., 58, 529-534.

Solomon, C.G. \& Dluhy, R.G. (2003) Re-thinking postmenopausal hormone therapy. N. Engl. J. Med., 348, 579-580.

van Dam, R.M., Schuit, A.J., Schouten, E.G., Vader, H.L. \& Pop. V.J. (2003) Serum cholesterol decline and depression in the postpartum period. J. Psychosom. Res., 46, 385-390.

Zweifel, J.E. \& O'Brien, W.H. (1997) A meta-analysis of the effect of hormone replacement therapy upon depressed mood. Psychoneuroendocrinology, 22, 189-212. 